\title{
Medical and Psychiatric Comorbidities in Korean Children and Adolescents with Attention-Deficit/Hyperactivity Disorder
}

\author{
Kee Jeong Park, Jung Sun Lee, and Hyo-Won Kim ${ }^{凶}$ \\ Department of Psychiatry, Asan Medical Center, University of Ulsan College of Medicine, Seoul, Republic of Korea
}

\begin{abstract}
Objective Attention-deficit/hyperactivity disorder (ADHD) is associated with a high rate of comorbid disorders. We aimed to investigate the medical and psychiatric comorbidities of Korean children and adolescents with ADHD.

Methods Data were obtained from Korean National Health Insurance Review and Assessment Service-National Patient Sample (HIRA-NPS) for 2011. We included 2,140 (mean age, 10.9 \pm 3.1 years; boys, 1,710) and 219,410 (non-ADHD; mean age, $12.4 \pm 3.7$ years; boys, $113,704)$ children and adolescents with and without ADHD, respectively. We compared medical and psychiatric comorbidities between the groups, and performed weighted logistic regression analyses to obtain odds ratios (ORs) and 95\% confidence intervals (CIs).

Results Most medical comorbidities were more likely in patients with ADHD and included nervous system disease (OR, 2.59; 95\% CI, 2.52-2.66); endocrine, nutritional, and metabolic disease (OR, 2.09; 95\% CI, 2.04-2.15); and congenital malformations, deformations, and chromosomal abnormalities (OR, 2.00; 95\% CI, 1.90-2.11). Oppositional defiant disorder and conduct disorder were more prevalent in patients with ADHD (OR, 81.88; 95\% CI, 79.00-84.86), followed by learning (OR, 75.61; 95\% CI, 69.69-82.04), and depressive disorders (OR, 55.76; 95\% CI, 54.44-57.11).

Conclusion Our results suggest that Korean children and adolescents with ADHD are more likely to suffer medical and psychiatric comorbidities than those without ADHD.

Psychiatry Investig 2017;14(6):817-824
\end{abstract}

Key Words Attention-deficit hyperactivity disorder, Comorbidity, Children, Adolescents.

\section{INTRODUCTION}

Attention-deficit/hyperactivity disorder (ADHD) is one of the most common childhood psychiatric disorders, with a prevalence rate of $5-8 \%$ in school-age children. It is characterized by symptoms of inattention, hyperactivity, and impulsivity ${ }^{1}$ that can lead to behavioral problems as well as educational and occupational underachievement. ${ }^{2}$

ADHD is associated with a high rate of comorbid psychiatric disorders. ${ }^{2}$ It has been reported that more than half of children and adolescents with ADHD experience comorbid psychiatric disorders, including oppositional defiant disorder (ODD) or conduct disorder (CD) ${ }^{3}$; anxiety disorder, depression,

Received: June 8, 2016 Revised: August 24, 2016

Accepted: December 5, 2016 Available online: August 25, 2017

$\triangle$ Correspondence: Hyo-Won Kim, MD, PhD

Department of Psychiatry, Asan Medical Center, University of Ulsan College of Medicine, 88 Olympic-ro 43-gil, Songpa-gu, Seoul 05505, Republic of Korea Tel: +82-2-3010-3414, Fax: +82-2-485-8381, E-mail: shingubi@amc.seoul.kr

(c) This is an Open Access article distributed under the terms of the Creative Commons Attribution Non-Commercial License (http://creativecommons.org/licenses/by$\mathrm{nc} / 4.0$ ) which permits unrestricted non-commercial use, distribution, and reproduction in any medium, provided the original work is properly cited. or dysthymia, ${ }^{4,5}$ and learning disorders. ${ }^{6}$ Studies from clinicreferred samples in both Western ${ }^{4,5}$ and Asian countries ${ }^{7,8}$ have reported ODD to be the most common comorbid disorder, followed by anxiety or other mood disorders. Further, community surveys in other countries have reported $\mathrm{CD}$, including ODD, to be the most common comorbid disorder in ADHD. ${ }^{3}$ In contrast, an epidemiological study in Korea reported that specific phobias were the most common comorbidity, followed by ODD and tic disorder. ${ }^{9}$ These comorbid psychiatric disorders have marked effects on the severity and impairment associated with ADHD symptoms, as well as on the long-term treatment response and outcomes in children and adolescents with ADHD. ${ }^{10}$ Therefore, it is crucial that we identify comorbid psychiatric disorders so that better intervention can be offered.

Beside psychiatric comorbidities, youths with ADHD are also more likely to have comorbid medical disorders. ${ }^{11}$ In addition, several medical conditions are known to be associated with ADHD, particularly neurological disorders such as epilepsy that directly influence the central nervous system. ${ }^{12}$ Other specific medical disorders, including strabismus, otitis me- 
dia, and chronic diseases of tonsils and adenoids (including adenotonsillar hypertrophy), may also cause attention problems and/or aggravate symptoms and functional impairments in those with pre-existing ADHD. ${ }^{13-16}$ Lastly, ADHD could cooccur with a childhood medical disorder, without there being a causative link; an example of this is asthma, which is one of the most common chronic childhood disorders. Nevertheless, it has been suggested that there is an increased risk of asthma in children with $\mathrm{ADHD}^{17}$ and that $\mathrm{ADHD}$ could be worsened by co-existing asthma. ${ }^{18}$ Indeed, impatience and impulsivity among subjects with ADHD could affect treatment adherence in chronic medical conditions and worsen outcomes. ${ }^{18}$ Appropriate management of these comorbid disorders could decrease the symptoms of $\mathrm{ADHD}$, and ADHD treatment could improve the outcome and prognosis of the medical disorder itself. ${ }^{16}$ Therefore, identifying medical and psychiatric comorbidities is important to ensure proper treatment and to improve the symptoms, functions, and quality of life of children with ADHD.

To date, although psychiatric comorbidities have been widely studied, there are only a few studies concerning medical comorbidities in patients with ADHD. Therefore, we aimed to investigate the medical and psychiatric comorbidities in Korean children and adolescents with ADHD using a nationwide population-based database.

\section{METHODS}

\section{Participants}

Korea has a single National Health Insurance program that covers $97 \%$ of the population. Health Insurance Review and Assessment (HIRA) covers all claims from National Health Insurance (97\%) and Medical Aid (other 3\%). HIRA database contains information pertaining to individual beneficiaries, including healthcare services such as diagnoses, procedures, and prescriptions. However, researchers have had problems accessing the database for research purposes considering its large size. To solve this problem, HIRA conducted a study to develop the National Patient Sample (NPS) using HIRA database in order to provide sample data for use in research. ${ }^{19}$ The HIRA-NPS database includes anonymous information concerning diagnoses based on the International Classification of Disease, 10th revision (ICD-10), medications prescribed, and hospital visits for approximately one million people. Data were extracted using random sampling methods and stratified by age and gender. The representativeness and validity of the sample was confirmed by comparing the estimation from the data and the whole population. ${ }^{19}$

The dataset analyzed by us comprised data from $1,375,842$ patients ( $3 \%$ of the total population) who received medical treatment from January 2011 to December 2011 (HIRA-NPS2011-0101). In 2011, there were 221,550 children and adolescents (age, 6-18 years) in the HIRA-NPS database. We subdivided these children and adolescents into two groups based on the ICD-10 (F90) classification of ADHD: those with ADHD and those without ADHD (non-ADHD).

\section{Medical and psychiatric diagnoses}

Medical conditions were categorized according to the Korean Standard Classification of Diseases, 6th revision (KCD-6). We compared medical comorbidities using chapters of KCD6. Chapters I-XVII relate to diseases and other morbid conditions, and chapter XIX relates to Injury, poisoning and certain other consequences of external causes. We excluded chapters which have no subjects in either the ADHD or the non-ADHD group: 'pregnancy, childbirth and the puerperium' and 'certain conditions originating in the perinatal period'. In addition, we chose five common medical disorders based on previous research, ${ }^{12-17}$ and these were categorized using three character categories from KCD-6, as follows: strabismus (ICD-10 codes: H49-50), otitis media (H65-67), chronic diseases of the tonsils and adenoids (J35), asthma (J45), and epilepsy (G40).

Psychiatric diagnoses were classified based on the ICD-10, as follows: intellectual disabilities (F70-79; 88), communication disorder (F80), autism spectrum disorder (F84), specific learning disorder (F81), tic disorder (F95), schizophrenia spectrum and other psychiatric disorders (F20-29), bipolar disorder (F30; 31), depressive disorder (F32; 33; 34.1) anxiety disorder (F40; 41; 43; 93.0; 94.0-94.2), somatoform disorder (F44; 45), obsessive compulsive disorder (F42), eating disorder (F50; 98.2; 98.3), ODD and CD (F91; 92), and sleep disorder (F51).

\section{Statistical analyses}

We compared demographic characteristic and the prevalence rates of comorbidities between the $\mathrm{ADHD}$ and non-ADHD groups. Chi-squared tests and independent t-tests were used for categorical and continuous variables, respectively. Weighted logistic regression analyses were performed, adjusting for age and gender to determine the odds of diagnoses in children with $\mathrm{ADHD}$ compared with non-ADHD. Odds ratios (ORs) and $95 \%$ confidence intervals (CIs) are reported. Statistical analyses were performed using R software (v2.15.3, R Foundation for Statistical Computing, Vienna, Austria).

\section{RESULTS}

\section{Participant characteristics}

Table 1 summarizes the demographic characteristics. The

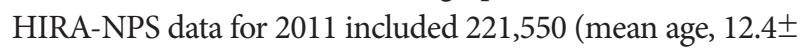
3.7 years; boys, 115,414) children and adolescents. Among 
them, 2,140 (mean age, 10.9 \pm 3.1 years; boys, 1,710) were diagnosed as $\mathrm{ADHD}$; $\mathrm{ADHD}$ prevalence was estimated to be $0.97 \%$. The mean age was younger in the ADHD group than in the non-ADHD group $(\mathrm{t}=19.48, \mathrm{p}<0.001)$. The gender distribution was also significantly different between the groups $\left(\chi^{2}=266.82\right.$, $\mathrm{p}<0.001)$. There were four-times as many boys as girls in the ADHD group.

\section{Comparison of the ADHD and non-ADHD groups by comorbidity}

\section{Comorbid medical disorders}

Most medical disorders were more frequent in the ADHD group than in the non-ADHD group. In the order of decreas- ing likelihood, the following comorbidities were present: nervous system disease (OR, 2.59; 95\% CI, 2.52-2.66); endocrine, nutritional, and metabolic disease (OR, 2.09; 95\% CI, 2.04-2.15); congenital malformations, deformations, and chromosomal abnormalities (OR, 2.00; 95\% CI, 1.90-2.11); disease of the circulatory system (OR, 1.79; 95\% CI, 1.71-1.87); disease of the blood and blood-forming organs and certain disorders of the immune mechanism (OR, 1.78; 95\% CI, 1.701.86); disease of the genitourinary system (OR, 1.66; 95\% CI, 1.61-1.71); disease of the eye and adnexa (OR, 1.46; 95\% CI, 1.44-1.48); disease of the ear and mastoid process (OR, 1.32; 95\% CI, 1.29-1.34); disease of the respiratory system (OR, 1.31; 95\% CI, 1.29-1.34); injury, poisoning, and other consequences of external causes (OR, 1.31; 95\% CI, 1.29-1.33); dis-

Table 1. Sample characteristics for youth with ADHD and youth without ADHD

\begin{tabular}{lccc}
\hline \multicolumn{1}{c}{ Characteristics } & $\begin{array}{c}\text { ADHD, N=2,140 } \\
\%(95 \% \mathrm{CI})\end{array}$ & $\begin{array}{c}\text { Non-ADHD, N=219,410 } \\
\%(95 \% \mathrm{CI})\end{array}$ & t or $\chi^{2}$ \\
\hline $\begin{array}{l}\text { Age }(\mathrm{yrs}) \\
6-11\end{array}$ & $58.87(58.67-59.08)$ & $41.39(41.19-41.60)$ & \\
12-18 & $41.12(40.92-41.33)$ & $58.61(58.40-58.81)$ & \\
Boys (yrs) & & & 266.82 \\
$6-11$ & $80.32(80.15-80.48)$ & $51.71(51.51-51.92)$ & $<0.001$ \\
$12-18$ & $79.32(79.15-79.49)$ & $51.90(51.69-52.11)$ & \\
\hline
\end{tabular}

ADHD: attention-deficit/hyperactivity disorder, CI: confidence interval

Table 2. Prevalence and odds ratios of medical comorbidities in youth with ADHD and without ADHD

\begin{tabular}{|c|c|c|c|}
\hline Medical comorbities & $\begin{array}{c}\text { ADHD } \\
\mathrm{N}=2,140 \\
\%(95 \% \mathrm{CI})\end{array}$ & $\begin{array}{c}\text { Non-ADHD } \\
\text { N=219,410 } \\
\%(95 \% \text { CI })\end{array}$ & $\begin{array}{l}\text { Odds ratios } \\
\quad 95 \% \mathrm{CI}\end{array}$ \\
\hline Certain infectious and parasitic diseases & $35.23(35.03-35.43)^{*}$ & $30.20(30.01-30.40)$ & $1.08(1.06-1.10)^{*}$ \\
\hline Neoplasm & $1.36(1.31-1.40)$ & $1.45(1.40-1.50)^{*}$ & $0.90(0.84-0.96)^{*}$ \\
\hline $\begin{array}{l}\text { Diseases of the blood and blood forming organs and certain } \\
\text { disorders involving the immune mechanism }\end{array}$ & $2.62(2.55-2.68)^{*}$ & $1.92(1.86-1.97)$ & $1.78(1.70-1.86)^{*}$ \\
\hline Endocrine, nutritional and metabolic diseases & $8.79(8.67-8.90)^{*}$ & $4.82(4.73-4.91)$ & $2.09(2.04-2.15)^{*}$ \\
\hline Diseases of the nervous systems & $8.36(8.25-8.48)^{*}$ & $4.06(3.97-4.14)$ & $2.59(2.52-2.66)^{*}$ \\
\hline Diseases of the eye and adnexa & $48.83(48.62-49.04)^{*}$ & $39.31(39.10-39.51)$ & $1.46(1.44-1.48)^{*}$ \\
\hline Diseases of the ear and mastoid process & $21.64(21.46-21.81)^{*}$ & $15.72(15.56-15.87)$ & $1.32(1.29-1.34)^{*}$ \\
\hline Diseases of the circulatory systems & $3.08(3.01-3.16)^{*}$ & $2.06(2.00-2.12)$ & $1.79(1.71-1.87)^{*}$ \\
\hline Diseases of the respiratory systems & $87.20(87.06-87.34)^{*}$ & $80.35(80.18-80.51)$ & $1.31(1.29-1.34)^{*}$ \\
\hline Diseases of the digestive systems & $85.56(85.41-85.71)^{*}$ & $81.44(81.28-81.60)$ & $1.23(1.20-1.26)^{*}$ \\
\hline Diseases of the skin and subcutaneous tissues & $44.11(43.90-44.32)^{*}$ & $37.83(37.63-38.04)$ & $1.24(1.22-1.26)^{*}$ \\
\hline Diseases of the musculoskeletal systems and connective tissues & $27.90(27.71-28.09)^{*}$ & $26.02(25.83-26.20)$ & $1.26(1.24-1.28)^{*}$ \\
\hline Diseases of the genitourinary systems & $6.68(6.58-6.79)^{*}$ & $5.91(5.81-6.01)$ & $1.66(1.61-1.71)^{*}$ \\
\hline $\begin{array}{l}\text { Congenital malformations, deformations and chromosomal } \\
\text { abnormalities }\end{array}$ & $2.06(2.00-2.12)^{*}$ & $0.91(0.87-0.94)$ & $2.00(1.90-2.11)^{*}$ \\
\hline $\begin{array}{l}\text { Injury, poisoning and certain other consequences of external } \\
\text { causes }\end{array}$ & $49.44(49.23-49.65)^{*}$ & $38.93(38.73-39.14)$ & $1.31(1.29-1.33)^{*}$ \\
\hline
\end{tabular}


ease of the musculoskeletal system and connective tissues (OR, 1.26; 95\% CI, 1.24-1.28); disease of the skin and subcutaneous tissues (OR, 1.24; 95\% CI, 1.22-1.26); disease of the digestive system (OR, 1.23; 95\% CI, 1.20-1.26); and certain infectious and parasitic diseases (OR, 1.08; 95\% CI, 1.06-1.10). Neoplasm (OR, 0.90; 95\% CI, 0.84-0.96) was the only disorder which was less prevalent in the ADHD group (Table 2).

Children and adolescents with ADHD more frequently had epilepsy compared with non-ADHD (OR, 6.42; 95\% CI, 6.166.70). Strabismus (OR, 1.79; 95\% CI, 1.72-1.85), chronic disease of the tonsils and adenoids (OR, 1.56; 95\% CI, 1.51-1.62), otitis media (OR, 1.23; 95\% CI, 1.21-1.26), and asthma (OR, 1.14 ; 95\% CI, 1.12-1.17) were also more common among children and adolescents with ADHD, but the ratio was less than two-fold (Table 3).

\section{Comorbid psychiatric disorders}

Most psychiatric comorbidities were frequent in children and adolescents with ADHD. When compared with non-AD$\mathrm{HD}, \mathrm{ODD}$ and CD were the most prevalent psychiatric comorbidities in the ADHD group (OR, 81.88; 95\% CI, 79.00-84.86). The following psychiatric comorbidities were present in the order of decreasing likelihood: specific learning disorder (OR, 75.61; 95\% CI, 69.69-82.04), depressive disorder (OR, 55.76; 95\% CI, 54.44-57.11), tic disorder (OR, 51.20; 95\% CI, 49.7552.68), bipolar disorder (OR, 50.10; 95\% CI, 47.56-52.76), communication disorder (OR, 32.83; 95\% CI, 30.80-35.01), intellectual disabilities (OR, 26.03; 95\% CI, 25.15-26.95), obsessive compulsive disorder (OR, 25.66; 95\% CI, 23.84-27.61), anxiety disorder (OR, 22.22; 95\% CI, 21.72-22.73), autism spectrum disorder (OR, 19.08; 95\% CI, 18.16-20.05), schizophrenia spectrum and other psychotic disorders (OR, 14.05;

Table 3. Prevalence of specific medical comorbidities in youth with ADHD and youth without ADHD

\begin{tabular}{lccc}
\hline \multicolumn{1}{c}{ Specific medical disorders } & ADHD, N=2,140 & Non-ADHD, N=219,410 & Odds ratios \\
$\%(95 \% \mathrm{CI})$ & $95 \% \mathrm{CI}$ & $6.42(6.16-6.70)^{*}$ \\
\hline Epilepsy & $3.36(3.29-3.44)^{*}$ & $0.54(0.51-0.57)$ & $1.79(1.72-1.85)^{*}$ \\
Strabismus & $4.16(4.08-4.24)^{*}$ & $1.98(1.92-2.04)$ & $1.23(1.21-1.26)^{*}$ \\
Otitis media & $13.13(12.99-13.27)^{*}$ & $8.93(8.81-9.05)$ & $1.56(1.51-1.62)^{*}$ \\
Chronic disease of tonsils and adenoids & $4.21(4.12-4.29)^{*}$ & $2.40(2.34-2.47)$ & $1.14(1.12-1.17)^{*}$ \\
Asthma & $18.27(18.11-18.43)^{*}$ & $13.00(12.82-13.11)$ & \\
\hline
\end{tabular}

All $\mathrm{p}$ value adjusted for age and gender. ${ }^{*}$ significant at the $\mathrm{p}=0.05$ level. ADHD: attention-deficit/hyperactivity disorder, CI: confidence interval

Table 4. Prevalence and odds ratios of psychiatric comorbidities in youth with ADHD and youth without ADHD

\begin{tabular}{|c|c|c|c|}
\hline Psychiatric comorbidities & $\begin{array}{c}\mathrm{ADHD}, \mathrm{N}=2,140 \\
\%(95 \% \mathrm{CI})\end{array}$ & $\begin{array}{c}\text { Non-ADHD, } \mathrm{N}=219,410 \\
\%(95 \% \mathrm{CI})\end{array}$ & $\begin{array}{l}\text { Odds ratios } \\
95 \% \mathrm{CI}\end{array}$ \\
\hline \multicolumn{4}{|l|}{ Neurodevelopmental disorders } \\
\hline Intellectual disabilities & $6.45(6.35-6.55)^{*}$ & $0.24(0.22-0.26)$ & $26.03(25.15-26.95)^{*}$ \\
\hline Communication disorder & $2.10(2.04-2.16)^{*}$ & $0.04(0.03-0.05)$ & $32.83(30.80-35.01)^{*}$ \\
\hline Autism spectrum disorder & $2.94(2.87-3.01)^{*}$ & $0.12(0.10-0.13)$ & $19.08(18.16-20.05)^{*}$ \\
\hline Specific learning disorder & $1.68(1.63-1.74)^{*}$ & $0.02(0.01-0.03)$ & $75.61(69.69-82.04)^{*}$ \\
\hline Tic disorder & $12.48(12.34-12.62)^{*}$ & $0.19(0.17-0.21)$ & $51.20(49.75-52.68)^{*}$ \\
\hline $\begin{array}{l}\text { Schizophrenia spectrum disorder and other } \\
\text { psychotic disorders }\end{array}$ & $1.03(0.99-1.07)^{*}$ & $0.10(0.09-0.12)$ & $14.05(12.99-15.19)^{*}$ \\
\hline \multicolumn{4}{|l|}{ Mood disorder } \\
\hline Bipolar disorder & $3.08(3.01-3.16)^{*}$ & $0.10(0.08-0.11)$ & $50.10(47.56-52.76)^{*}$ \\
\hline Depressive disorder & $17.15(17.00-17.31)^{*}$ & $0.60(0.57-0.63)$ & $55.76(54.44-57.11)^{*}$ \\
\hline Anxiety disorder & $15.28(15.13-15.43)^{*}$ & $1.19(1.14-1.23)$ & $22.22(21.72-22.73)^{*}$ \\
\hline Obsessive compulsive disorder & $1.31(1.26-1.36)^{*}$ & $0.07(0.06-0.08)$ & $25.66(23.84-27.61)^{*}$ \\
\hline Somatoform disorder & $1.17(1.12 .-1.21)^{*}$ & $0.54(0.51-0.57)$ & $3.02(2.82-3.24)^{*}$ \\
\hline Eating disorder & $0.37(0.35-0.40)^{*}$ & $0.04(0.03-0.05)$ & $11.17(9.82-12.71)^{*}$ \\
\hline Sleep disorder & $1.26(1.21-1.31)^{*}$ & $0.15(0.13-0.16)$ & $13.92(12.97-14.93)^{*}$ \\
\hline $\mathrm{ODD} / \mathrm{CD}$ & $8.74(8.62-8.86)^{*}$ & $0.12(0.10-0.13)$ & $81.88(79.00-84.86)^{*}$ \\
\hline
\end{tabular}

All $\mathrm{p}$ value adjusted for age and gender. *significant at the $\mathrm{p}=0.05$ level. ADHD: attention-deficit/hyperactivity disorder, CD: conduct disorder, CI: confidence interval, ODD: oppositional defiant disorder 
95\% CI, 12.99-15.19), sleep disorder (OR, 13.92; 95\% CI, 12.9714.93), eating disorder (OR, 11.17; 95\% CI, 9.82-12.71), and somatoform disorder (OR, 3.02; 95\% CI, 2.82-3.24) (Table 4).

\section{DISCUSSION}

In this study, most medical and psychiatric comorbidities were more frequent in the ADHD group than in the non-ADHD group. To the best of our knowledge, this is the only study to investigate medical and psychiatric comorbidities of ADHD in children and adolescents using a nationally representative sample.

We found that ADHD prevalence was $0.97 \%$ in our national insurance database, which is much lower than that reported by epidemiological studies from Western countries ${ }^{20,21}$ and by other studies from Korea. ${ }^{9}$ The worldwide prevalence of ADHD in children and adolescents has been reported to be $2.2-17.8 \%$ in epidemiological studies ${ }^{20}$ and $5.29 \%$ in a meta-analysis. ${ }^{21}$ Previous researchers have suggested that this wide variation in prevalence rates is reflective of methodological rather than cultural differences. ${ }^{21}$ In contrast to the previous epidemiological study from Korea, which estimated the prevalence of ADHD to be $5.9 \%,{ }^{9}$ our data were based on patients who received medical treatment and reflect prevalence of ADHD medication rather than real prevalence. Similarly, the prevalence rate was reported to be as low as $1.64 \%$ with medically treated sample using national populationbased data in Taiwan. ${ }^{22}$ The Taiwanese prevalence suggested that over $50 \%$ of children who was diagnosed with ADHD did not receive treatment ${ }^{22}$ and this result is consistent with experiences in Western countries. ${ }^{23}$ In addition, we defined ADHD based on ICD-10. Because the ICD-10 definition of Hyperkinetic Disorder is a narrower classification equivalent only to the ADHD combined subtype, this could be another reason for the low ADHD prevalence rate in our study. ${ }^{21}$ However, our results still suggest that the treatment rate in Korea is much lower than in either Taiwan or Western countries. Accordingly, early detection and treatment need to be improved in Korea.

In this study, ODD or CD was the most common comorbidities associated with ADHD in children and adolescents, which is consistent with the findings of previous studies. ${ }^{5,24}$ In our study, although the rate of comorbid ODD or CD $(8.74 \%)$ is much lower than that reported by previous studies (40-84\%), ${ }^{4,25}$ OR for ODD and CD (81.88) is much higher than that reported in Angold's meta-analysis $(10.7)^{26}$ or in a previous Korean community-based study (9.90 and 22.31 for ODD and CD, respectively). ${ }^{19}$ We classified ADHD based on the ICD-10 (F90). Therefore, those who both met criteria for ADHD and CD/ ODD (F90.1, hyperkinetic conduct disorder) were included in ADHD group not ADHD comorbid with CD/ODD in this study. This might explain that the prevalence rates of ODD and CD are lower than previous studies. ${ }^{4,25}$ The relationship between ODD and ADHD is a controversial issue. Several studies have found that ODD shares cognitive impairments, social impairments, and behavioral problems with $\mathrm{ADHD},{ }^{27,28}$ whereas others have found that ODD could be more clearly differentiated from ADHD on the basis of clinical or psychosocial characteristics. ${ }^{29,30}$

The second most prevalent comorbid disorder was specific learning disorder. Previous studies have reported that learning disorder is comorbid with ADHD in $20-30 \%$ children and that $\mathrm{ADHD}$ is comorbid with learning disorder in 9-60\% children. ${ }^{2,6,31}$ We found a much lower comorbidity rate of specific learning disorder than that previously reported. Because treatment for learning disorder is not covered by National Health Insurance, learning disorder tends to be diagnosed more in educational settings than in clinics. This could contribute to the low prevalence of learning disorder in our study. However, the OR was 75.61 for having a learning disorder. In addition to inattention, comorbid learning disorder could contribute to the poor academic performance in children with ADHD. Although there are still controversies surrounding the relationship between ADHD and learning disorders, evidence from genetic and neurobiological studies imply that learning disorders, particularly dyslexia, share common pathophysiological pathways. Moreover, ADHD and dyslexia share neuropsychological profiles in attention and response inhibition, processing speed, and working memory. ${ }^{31}$

In our study, mood and anxiety disorders were more prevalent in the ADHD group. Children and adolescents with ADHD have been shown to have a higher likelihood of having comorbid mood and anxiety disorders in previous clinicreferred samples and meta-analysis ${ }^{5,26}$; this finding is consistent with our results. However, compared to previous studies, we showed that there was a much higher OR for comorbid bipolar disorder. Concerning the relationship between ADHD and internalizing disorders, several studies have found that depressive disorders share genetic components with ADHD, whereas anxiety disorders do not. ${ }^{6}$ Besides, difficulties in regulating emotions, high levels of parent-child conflict, poor academic performance, and peer problems among youths with ADHD could influence the occurrence of comorbid internalizing disorders. ${ }^{32,33}$ Consequently, the relationship between $\mathrm{ADHD}$ and bipolar disorder appears to be more complex than previously thought. Indeed, while some researchers have suggested that ADHD could be a prodromal manifestation of pediatric-onset bipolar disorder and that it may share a common biological etiology, ${ }^{34}$ other experts have pointed out possibility of overdiagnosis of ADHD as a bipolar disorder or as 
a comorbid disorder. ${ }^{26}$

Consistent with previous research, we found that most medical disorders were more prevalent in children and adolescents with ADHD. ${ }^{11}$ Although there is limited literature pertaining to comorbidities in $\mathrm{ADHD}$, some researchers have attempted to explain the reason why ADHD is associated with several general medical disorders. They have suggested that a diminished concern for health-conscious behavior in $\mathrm{ADHD}$ is a possible cause, ${ }^{35}$ but this does not completely explain the higher incidence of general medical disorders in ADHD.

Neoplasm was the only disorder which was less prevalent in the ADHD group. ADHD had a reported prevalence of 9.0$10.5 \%$ in survivors of childhood leukemia or brain tumor. ${ }^{36,37}$ These rates are similar to those in the general population. However, another study reported that the prevalence of ADHD in childhood cancer may be lower than in the general popula$\operatorname{tion}^{38}$ for several reasons. First, it may be difficult to distinguish symptoms of ADHD from treatment sequelae of childhood cancer. After cranial radiation therapy or intrathecal chemotherapy for childhood cancer, clinically significant deficits in attention, processing speed and executive function were reported, ${ }^{39}$ and these deficits overlap with core neurocognitive deficits of ADHD. Second, parents have paid less attention to cognitive impairment, especially ADHD, in children with cancer compared to the physical symptoms and emotional and/or behavioral problems, ${ }^{40}$ and relatively little research has been conducted on cognitive problems including ADHD in childhood cancer. ${ }^{41}$ Further studies are needed to better understand the relationship between ADHD and neoplasm in children and adolescents.

$\mathrm{ADHD}$ as a comorbidity of other medical disorders has been more frequently studied than have medical disorders as comorbidities of ADHD. Previous studies have shown that ADHD might be associated with specific medical disorders such as otitis media, strabismus, chronic disease of the tonsils and adenoids (including adenotonsillar hypertrophy), and asthma. ${ }^{2,11}$ Otitis media and adenotonsillar hypertrophy are related to attention deficiency, and their treatment can improve attention problems. ${ }^{42}$ Similar to the results of this study, higher rates of strabismus have been reported in previous studies of $\mathrm{ADHD},{ }^{14}$ but the causal relationship between $\mathrm{ADHD}$ and strabismus has not been revealed. These studies implied that strabismus is a manifestation of immaturities in the visuomotor, spatial, and attention processing mechanisms. ${ }^{43}$ In addition, it is known that asthma can lead to emotional disturbances, low school participation and performance, ${ }^{44}$ and psychosocial problems, which could negatively influence preexisting ADHD symptoms. However, there are few studies on the relationship between ADHD and these specific disorders; further research is needed to identify the presence of a causal link, if any.

Many children with epilepsy report attention problems, and one-third have a diagnosis of ADHD. ${ }^{45}$ Our results are consistent with these previous findings of a high prevalence of comorbid ADHD with epilepsy. ${ }^{45,46}$ The high incidence of abnormalities on electroencephalography in ADHD, such as epileptiform discharges, also implicates a pathophysiological overlap between ADHD and epilepsy. ${ }^{47}$ Furthermore, frontostriatal network dysfunction is known to be associated with $\mathrm{ADHD}$, and epilepsy, which disrupts this network, also manifests similar symptoms to ADHD. ${ }^{48}$ Several researchers have supported this by reporting that ADHD, particularly the inattentive subtype, is often associated with epilepsy. ${ }^{12,49}$ Identifying and managing attention deficit or ADHD in children with epilepsy is important for improving academic achievement and for formation and maintenance of interpersonal relationships. ${ }^{50}$

Some limitations should be considered when interpreting our findings. First, this study used data from the HIRA-NPS database. Because the HIRA-NPS data is from a clinically referred population, cautious interpretation is needed regarding referral bias. Moreover, some conditions may be missing from the HIRA-NPS data because the HIRA-NPS data only includes information about reimbursable medical utilization. Second, the diagnosis of ADHD and comorbid psychiatric disorders was not based on structured interviews. Further, the diagnosis of specific learning disorder was not confirmed by individually administered standardized achievement measures. Clinical diagnoses tend to be specific, with fewer false positives, but they may be less sensitive to detecting comorbid conditions, and often identify fewer total diagnoses than would result from structured or semi-structured interviews. ${ }^{51}$ Third, it is difficult to establish causality because this was a cross-sectional population-based study. Fourth, for the same reason, it is unclear whether the medical disorder was diagnosed first and ADHD is a comorbidity of the medical disorder or ADHD was diagnosed earlier. Subjects who have medical disorders and are receiving treatment may have a greater chance of early detection and referral for ADHD treatment than physically healthy subjects. Fifth, we could not track the site where the diagnosis is made. Because medical clinics are less likely to make psychiatric diagnoses and psychiatric clinics are less likely to make medical diagnoses, the comorbidity rates might be affected by the site. Sixth, because individuals in the HIRA-NPS database were seeking treatment, rates of ADHD and comorbidities could have been influenced by factors such as severity of presentation and attitudes toward treatment. ${ }^{52}$ Seventh, although age and gender were adjusted in weighted logistic regression analysis, other confounding variables such as socioeconomic status have not been taken into account. 
Eighth, we presented limited demographic characteristics of subjects, because the HIRA dataset doesn't include more detailed information such as educational levels, parental information and socioeconomic status which could be important to understand characteristics of the subjects. Lastly, we did not differentiate between children and adolescents, which is important considering previous studies have indicated that these groups have different rates of comorbidities. ${ }^{8}$

Despite these caveat, this study suggests that children and adolescents with ADHD have more medical and psychiatric comorbidities than those without ADHD in Korea.

\section{Acknowledgments}

This research was supported by the Basic Science Research Program through the National Research Foundation of Korea (NRF) funded by the Ministry of Science, ICT \& Future Planning (2015R1A1A3A04001349).

\section{REFERENCES}

1. American Psychiatric Association. Diagnostic and Statistical Manual for Mental Disorder IV (DSM-IV). Washington DC: American Psychiatric Association; 1994.

2. Barkley RA. Attention Deficit Hyperactivity Disorder: A Handbook for Diagnosis and Treatment. New York: Guilford Press; 2006.

3. Bird HR, Gould MS, Staghezza BM. Patterns of diagnostic comorbidity in a community sample of children aged 9 through 16 years. J Am Acad Child Adolesc Psychiatry 1993;32:361-368.

4. Jensen PS, Hinshaw SP, Kraemer HC, Lenora N, Newcorn JH, Abikoff $\mathrm{HB}$, et al. ADHD comorbidity findings from the MTA study: comparing comorbid subgroups. J Am Acad Child Adolesc Psychiatry 2001;40: 147-158.

5. Wilens TE, Biederman J, Brown S, Tanguay S, Monuteaux MC, Blake $\mathrm{C}$, et al. Psychiatric comorbidity and functioning in clinically referred preschool children and school-age youths with ADHD. J Am Acad Child Adolesc Psychiatry 2002;41:262-268.

6. Pliszka SR. Comorbidity of attention-deficit/hyperactivity disorder with psychiatric disorder: an overview. J Clin Psychiatry 1998;59(Suppl 7): 50-58.

7. Byun H, Yang J, Lee M, Jang W, Yang JW, Kim JH, et al. Psychiatric comorbidity in Korean children and adolescents with attention-deficit hyperactivity disorder: psychopathology according to subtype. Yonsei Med J 2006;47:113-121.

8. Huh Y, Choi I, Song M, Kim S, Hong SD, Joung Y. A comparison of comorbidity and psychological outcomes in children and adolescents with attention-deficit/hyperactivity disorder. Psychiatry Investig 2011;8: 95-101.

9. Cho SC, Kim BN, Kim JW, Rohde LA, Hwang JW, Chungh DS, et al. Full syndrome and subthreshold attention-deficit/hyperactivity disorder in a Korean community sample: comorbidity and temperament findings. Eur Child Adolesc Psychiatry 2009;18:447-457.

10. Hechtman L. Assessment and diagnosis of attention-deficit/hyperactivity disorder. Child Adolesc Psychiatr Clin N Am 2000;9:481-498.

11. Schieve LA, Gonzalez V, Boulet SL, Visser SN, Rice CE, Van Naarden Braun $\mathrm{K}$, et al. Concurrent medical conditions and health care use and needs among children with learning and behavioral developmental disabilities, National Health Interview Survey, 2006-2010. Res Dev Disabil 2012;33:467-476.

12. Hesdorffer DC, Ludvigsson P, Olafsson E, Gudmundsson G, Kjartansson $\mathrm{O}$, Hauser WA. ADHD as a risk factor for incident unprovoked seizures and epilepsy in children. Arch Gen Psychiatry 2004;61:731-736.

13. Jonsson P, Jonsson B, Eeg-Olofsson O. Psychological and social outcome of epilepsy in well-functioning children and adolescents. A 10-year follow-up study. Eur J Paediatr Neurol 2014;18:381-390.

14. Granet DB, Gomi CF, Ventura R, Miller-Scholte A. The relationship between convergence insufficiency and ADHD. Strabismus 2005;13:163168.

15. Bennett KE, Haggard MP, Silva PA, Stewart IA. Behaviour and developmental effects of otitis media with effusion into the teens. Arch Dis Child 2001;85:91-95.

16. Soylu E, Soylu N, Yildirim YS, Sakallioglu O, Polat C, Orhan I. Psychiatric disorders and symptoms severity in patients with adenotonsillar hypertrophy before and after adenotonsillectomy. Int J Pediatr Otorhinolaryngol 2013;77:1775-1781.

17. Leibson CL, Katusic SK, Barbaresi WJ, Ransom J, O'Brien PC. Use and costs of medical care for children and adolescents with and without attention-deficit/hyperactivity disorder. JAMA 2001;285:60-66.

18. Nylander C, Fernell E, Tindberg Y. Chronic conditions and coexisting ADHD-a complicated combination in adolescents. Eur J Pediatr 2015; 174:1209-1215.

19. Kim L, Sakong J, Kim Y, Kim S, Kim S, Tchoe B, et al. Developing the inpatient sample for the national health insurance claims data. Health Pol Manag 2013;23:152-161.

20. Skounti M, Philalithis A, Galanakis E. Variations in prevalence of attention deficit hyperactivity disorder worldwide. Eur J Pediatr 2007;166: 117-123.

21. Polanczyk G, de Lima MS, Horta BL, Biederman J, Rohde LA. The worldwide prevalence of ADHD: a systematic review and metaregression analysis. Am J Psychiatry 2007;164:942-948.

22. Chien IC, Lin CH, Chou YJ, Chou P. Prevalence, incidence, and stimulant use of attention-deficit hyperactivity disorder in Taiwan, 19962005: a national population-based study. Soc Psychiatry Psychiatr Epidemiol 2012;47:1885-1890.

23. Hechtman L. Long-term treatment of children and adolescents with attention-deficit/hyperactivity disorder (ADHD). Curr Psychiatry Rep 2006;8:398-408.

24. Keenan K, Wakschlag LS. More than the terrible twos: the nature and severity of behavior problems in clinic-referred preschool children. J Abnorm Child Psychol 2000;28:33-46.

25. Barkley RA. Attention Deficit Hyperactivity Disorder: A Clinical Handbook. New York: Guilford; 2005.

26. Angold A, Costello EJ, Erkanli A. Comorbidity. J Child Psychol Psychiatry 1999;40:57-87.

27. Oosterlaan J, Scheres A, Sergeant JA. Which executive functioning deficits are associated with $\mathrm{AD} / \mathrm{HD}, \mathrm{ODD} / \mathrm{CD}$ and comorbid AD/HD+ ODD/CD? J Abnorm Child Psychol 2005;33:69-85.

28. Stevenson J, Asherson P, Hay D, Levy F, Swanson J, Thapar A, et al. Characterizing the ADHD phenotype for genetic studies. Dev Sci 2005; 8:115-121.

29. Drabick DA, Gadow KD, Carlson GA, Bromet EJ. ODD and ADHD symptoms in Ukrainian children: external validators and comorbidity. J Am Acad Child Adolesc Psychiatry 2004;43:735-743.

30. Kim HW, Cho SC, Kim BN, Kim JW, Shin MS, Yeo JY. Does oppositional defiant disorder have temperament and psychopathological profiles independent of attention deficit/hyperactivity disorder? Compr Psychiatry 2010;51:412-418.

31. Sexton CC, Gelhorn HL, Bell JA, Classi PM. The co-occurrence of reading disorder and ADHD: epidemiology, treatment, psychosocial impact, and economic burden. J Learn Disabil 2012;45:538-564.

32. Meinzer MC, Pettit JW, Viswesvaran C. The co-occurrence of attentiondeficit/hyperactivity disorder and unipolar depression in children and adolescents: a meta-analytic review. Clin Psychol Rev 2014;34:595-607.

33. Marmorstein NR. Relationships between anxiety and externalizing disorders in youth: the influences of age and gender. J Anxiety Disord 2007;21:420-432.

34. Jerrell JM, McIntyre RS, Park YM. Correlates of incident bipolar disorder in children and adolescents diagnosed with attention-deficit/hyper- 
activity disorder. J Clin Psychiatry 2014;75:e1278-e1283.

35. Barkley RA, Murphy KR, Fischer M. ADHD in adults: What the Science Says. New York: Guilford Press; 2010.

36. Krull KR, Khan RB, Ness KK, Ledet D, Zhu L, Pui CH, et al. Symptoms of attention-deficit/hyperactivity disorder in long-term survivors of childhood leukemia. Pediatr Blood Cancer 2011;57:1191-1196.

37. Kahalley LS, Conklin HM, Tyc VL, Wilson SJ, Hinds PS, Wu S, et al. $\mathrm{ADHD}$ and secondary ADHD criteria fail to identify many at-risk survivors of pediatric ALL and brain tumor. Pediatr Blood Cancer 2011; 57:110-118.

38. Haddy TB, Mosher RB, Reaman GH. Late effects in long-term survivors after treatment for childhood acute leukemia. Clin Pediatr (Phila) 2009; 48:601-608.

39. Mulhern RK, Butler RW. Neurocognitive sequelae of childhood cancers and their treatment. Pediatr Rehabil 2004;7:1-14; discussion 15-16.

40. Yeh CH, Hung LC, Chao KY. The quality of life for cancer children (QOLCC) for Taiwanese children with cancer (part II): feasibility, crossinformants variance and clinical validity. Psychooncology 2004;13:171176.

41. Briere ME, Scott JG, McNall-Knapp RY, Adams RL. Cognitive outcome in pediatric brain tumor survivors: delayed attention deficit at longterm follow-up. Pediatr Blood Cancer 2008;50:337-340.

42. Ayral M, Baylan MY, Kinis V, Bez Y, Bakir S, Ozbay M, et al. Evaluation of hyperactivity, attention deficit, and impulsivity before and after adenoidectomy/adenotonsillectomy surgery. J Craniofac Surg 2013;24: 731-734.

43. Borsting E, Rouse M, Chu R. Measuring ADHD behaviors in children with symptomatic accommodative dysfunction or convergence insufficiency: a preliminary study. Optometry 2005;76:588-592.

44. Yuksel H, Sogut A, Yilmaz O. Attention deficit and hyperactivity symptoms in children with asthma. J Asthma 2008;45:545-547.

45. Dunn DW, Austin JK, Harezlak J, Ambrosius WT. ADHD and epilepsy in childhood. Dev Med Child Neurol 2003;45:50-54.

46. Semrud-Clikeman M, Wical B. Components of attention in children with complex partial seizures with and without ADHD. Epilepsia 1999; 40:211-215.

47. Lee EH, Choi YS, Yoon HS, Bahn GH. Clinical impact of epileptiform discharge in children with attention-deficit/hyperactivity disorder (ADHD). J Child Neurol 2016;31:584-588.

48. Koneski JA, Casella EB. Attention deficit and hyperactivity disorder in people with epilepsy: diagnosis and implications to the treatment. Arq Neuropsiquiatr 2010;68:107-114.

49. Socanski D, Aurlien D, Herigstad A, Thomsen PH, Larsen TK. Epilepsy in a large cohort of children diagnosed with attention deficit/hyperactivity disorders (ADHD). Seizure 2013;22:651-655.

50. Caplan R, Sagun J, Siddarth P, Gurbani S, Koh S, Gowrinathan R, et al. Social competence in pediatric epilepsy: insights into underlying mechanisms. Epilepsy Behav 2005;6:218-228.

51. Garb HN. Studying the Clinician: Judgment Research and Psychological Assessment. Washington, DC: American Psychological Association; 1998.

52. Berkson J. Limitations of the application of fourfold table analysis to hospital data. Biometrics 1946;2:47-53. 